\title{
The First Hypertension Trial Comparing the Effects of Two Fixed-Dose Combination Therapy Regimens on Cardiovascular Events: Avoiding Cardiovascular Events Through Combination Therapy in Patients Living With Systolic Hypertension (ACCOMPLISH)
}

Kenneth A. Jamerson, MD

Current recommendations for the treatment of bypertension clarify the need to achieve lower blood pressure levels in the general population ( $<140 / 90 \mathrm{~mm} \mathrm{Hg}$ ) and in specific high-risk patient groups such as patients with diabetes or chronic renal disease $(<130 / 80 \mathrm{~mm} \mathrm{Hg})$. Further, it is evident that to reach appropriate blood pressure control, most patients with high blood pressure will require two or more antihypertensive

From the Department of Internal Medicine, University of Michigan Medical Center,

Ann Arbor, MI

Address for correspondence:

Kenneth A. Jamerson, MD, Professor of Internal Medicine, Division of Cardiovascular Medicine, Department of Internal Medicine, University of Michigan Medical Center, 3918 Taubman

Center, Ann Arbor, MI 48109

E-mail:jamerson@umich.edu

ID: 2676 agents. There is scarce clinical trial outcome evidence to guide clinicians in the selection of optimal combinations of antibypertensive classes for high-risk bypertensive patients. A new clinical trial, Avoiding Cardiovascular Events through Combination Therapy in Patients Living with Systolic Hypertension (ACCOMPLISH), is the first large clinical trial to directly compare cardiovascular mortality and morbidity rates for two fixed-dose combination therapies. Results from ACCOMPLISH should provide muchneeded guidance for selecting optimal combination therapy for high-risk hypertensive patients. (J Clin Hypertens. 2003;5(4 suppl 3):29-35)

๑2003 Le Jacq Communications, Inc.

The majority of evidence demonstrating 1 that lowering blood pressure reduces the risk of cardiovascular disease (CVD) events is derived from clinical trials of diureticbased or $\beta$ blocker-based therapies, ${ }^{1}$ and 
clinical recommendations endorsed these two classes of agents as initial treatment for hypertension. ${ }^{2,3}$ The most current recommendations now suggest that blood pressure levels recently labeled "normal" may represent "prehypertension" due to their association with increased CVD risk. ${ }^{4}$ After early trials with diuretics and $\beta$ blockers established that lowering elevated blood pressure improved outcomes, researchers set out to determine if other antihypertensive therapies might reduce events still further. ${ }^{5}$ Before 1999, no published mortality/morbidity trial had compared the effects of newer antihypertensive medications (such as angiotensinconverting enzyme [ACE] inhibitors, calcium channel blockers [CCBs], and $\alpha$ blockers) with diuretics and $\beta$ blockers in the treatment of essential hypertension. ${ }^{6}$ In the past 10 years, ACE inhibitors and CCBs have demonstrated efficacy at reducing CVD morbidity and mortality compared with placebo in a wide range of high-risk patient groups. ${ }^{4}$ In comparative trials with these agents and diuretic/ $\beta$ blockers, equivalent reductions in cardiovascular events have been noted. ${ }^{5}$ The antihypertensive class of angiotensin II receptor blockers (ARBs) has demonstrated the ability to lower blood pressure, and clinical trial data demonstrating the cardioprotective effects of ARBs are emerging. The recent Losartan Intervention for Endpoint Reduction in Hypertension $\left(\right.$ LIFE) ${ }^{7}$ trial was the first large study to actually show superiority in primary clinical outcomes (primarily strokes) for a newer agent, the ARB losartan, compared with an older agent, the $\beta$ blocker atenolol (Figure 1).

Despite an enormous amount of accumulated trial data, there is still debate about which agent as monotherapy, or which combination regimen, is superior in reducing overall CVD morbidity and mortality or total mortality. This lack of evidence does not prove or disprove superiority of some classes compared to others; instead, it reflects limitations in the trial designs, ${ }^{6-14}$ which prevent the detection of differences that may exist. The Antihypertensive and Lipid-Lowering Treatment to Prevent Heart Attack Trial (ALLHAT) ${ }^{8}$ is an important

\begin{tabular}{|c|c|c|c|c|}
\hline Trial & $N$ & $\begin{array}{c}\text { Primary end point } \\
\text { Relative risk ( } 95 \% \mathrm{Cl})\end{array}$ & Comparator & $p$ value \\
\hline CAPPP & 10,985 & $\mapsto-1$ & captopril & 0.52 \\
\hline STOP-2 & 6,614 & $\mapsto-1$ & $A C E I / C C B$ & 0.89 \\
\hline NORDIL & 10,984 & $\mapsto-1$ & diltiazem & 0.97 \\
\hline INSIGHT & 6,321 & $\longmapsto-1$ & nifedipine GITS & 0.34 \\
\hline \multirow[t]{2}{*}{ ALLHAT } & 33,357 & $\mapsto-1$ & amlodipine besylat & te 0.65 \\
\hline & & $1-1$ & lisinopril & 0.81 \\
\hline CONVINCE & 16,606 & $\mapsto-1$ & verapamil-COER & 0.77 \\
\hline \multirow[t]{3}{*}{ LIFE } & 9,193 & $|\mapsto|$ & losartan & 0.02 \\
\hline & & $\begin{array}{lllllll}0.4 & 0.6 & 0.8 & 1.0 & 1.2 & 1.4 & 1\end{array}$ & & \\
\hline & Favors diut & etics/B blockers & newer agents & \\
\hline
\end{tabular}

Figure 1. Recent hypertension trial results comparing newer antihypertensive agents with older agents (as noted) for the primary end point (composite of cardiovascular disease [CVD] death, myocardial infarction [MI], or stroke, or as noted below) for each trial. The Losartan Intervention for Endpoint Reduction in Hypertension (LIFE) study is the only trial that has shown a significant advantage for an agent from a newer class (angiotensin II receptor blockers [ARBs]), when compared with an older agent. Captopril Prevention Project randomized trial (CAPPP) (captopril $v$ s. diuretic and $\beta$ blocker); Swedish Trial in Old Patients with Hypertension-2 (STOP-2) (angiotensinconverting enzyme inhibitor or calcium channel blocker vs. conventional treatment; composite primary end point: fatal stroke, fatal MI, or other fatal CVD event); Nordic Diltiazem Study (NORDIL) (diltiazem vs. diuretic and $\beta$ blocker); International Nifedipine GITS study: Intervention as a Goal in Hypertension Treatment (INSIGHT) (nifedipine vs. bydrochlorothiazide-amiloride; composite primary end point: CVD death, MI, stroke, or heart failure); Antibypertensive and Lipid-Lowering Treatment to Prevent Heart Attack Trial (ALLHAT) (chlorthalidone vs. lisinopril or amlodipine besylate); Controlled Onset Verapamil Investigation of Cardiovascular Endpoints study (CONVINCE) (controlled onset extended-release verapamil vs. atenolol or hydrochlorothiazide); LIFE (atenolol vs. losartan). ${ }^{6-14}$ $C I=$ confidence interval; GITS=gastrointestinal therapeutic system

case in point. This large study, sponsored by the National Heart, Lung and Blood Institute, showed no difference among three major antihypertensive classes (diuretics, ACE inhibitors, and CCBs) for the primary clinical outcome. However, there were fewer episodes of congestive heart failure in the diuretic compared with the amlodipine group and fewer strokes and congestive heart failure cases compared with patients on lisinopril. However, less than $50 \%$ of patients in the ALLHAT trial achieved blood pressure control on monotherapy. Thus, 
the ALLHAT trial investigated a strategy of controlling blood pressure with a regimen that began with a diuretic, an ACE inhibitor, or a CCB but in most cases required add-on therapy. The superiority of any specific monotherapy may have been obscured. Moreover, the study design forced together combinations that clinicians seldom choose. As in recent clinical trials, the need for combination therapy to achieve blood pressure control has hampered investigators in their efforts to delineate clear differences among classes of agents.

A clear message has emerged from the era of clinical trials that attempted to determine the superiority of specific drug classes: Lowering blood pressure imparts cardioprotection; the specific agent used to achieve control may have less importance. Frequently, multiple drug classes are needed to achieve control. The recently published seventh report of the Joint National Committee on Prevention, Detection, Evaluation, and Treatment of High Blood Pressure (JNC 7) suggests that patients with a systolic blood pressure $20 \mathrm{~mm} \mathrm{Hg}$ or more above goal receive combination therapy (rather than monotherapy) as the initial strategy for controlling blood pressure. Moreover, JNC 7 recommends a diuretic as part of the combination strategy. While this makes empirical sense, there are no clinical trial data demonstrating the clinical efficacy of initial combination therapy, nor is there much evidence to support any particular combination of drug classes. It appears that a new paradigm is needed for designing hypertension trials in the 21 st century. This article describes the Avoiding Cardiovascular Events through Combination Therapy in Patients Living with Systolic Hypertension (ACCOMPLISH) trial, ${ }^{15}$ the first large, randomized clinical trial to directly compare CVD mortality and morbidity rates for two preselected combination therapies.

\section{THE NEW ERA:}

\section{COMBINATION THERAPY}

Most patients with hypertension require combination antihypertensive therapy, making this a better strategy for controlling blood pressure compared with monotherapy. Even high-dose monotherapy is generally less successful than low doses of agents from two separate classes for blood pressure control and tolerability. ${ }^{16} \mathrm{It}$ is possible that specific combinations of agents could improve CVD outcomes by targeting more than a single pathway in the pathology of hypertension. This benefit, if proven, would be of particular relevance in the treatment of high-risk patients. However, combination therapy has not previously been evaluated in a randomized clinical trial as a strategy for achieving blood pressure control and preventing CVD events in high-risk patients.

The ACCOMPLISH study ${ }^{15}$ is a large outcomes trial that will randomize subjects to a specific fixed-dose combination agent as initial therapy. This ongoing trial will evaluate whether the fixed-dose combination of an ACE inhibitor/CCB (amlodipine besylate/benazepril $\mathrm{HCl}$ ) provides superior benefits in reducing morbidity and mortality from CVD events in a high-risk hypertensive population when compared with an ACE inhibitor/diuretic combination (benazepril $\mathrm{HCl} /$ hydrochlorothiazide).

\section{The Role for ACE Inhibitors in \\ High-Risk Patients}

ACE inhibitors will be included in the regimens of both groups of participants in ACCOMPLISH because of their proven benefits in a wide range of high-risk patients. The class of ACE inhibitors has been used for lowering blood pressure for more than 20 years and has been shown to be beneficial in elderly individuals ${ }^{17}$ and in patients with heart failure, myocardial infarction (MI), diabetes, diabetic and nondiabetic chronic renal disease, and atherosclerotic vascular disease. ${ }^{4,18-22}$ ACE inhibitors have demonstrated the ability to reduce the incidence of cardiac death, nonfatal MI, stroke, end-stage renal disease, and complications of heart failure. ${ }^{4,18,22}$ They have also been shown to improve insulin resistance in patients with metabolic syndrome; reduce CVD events in patients with diabetes ${ }^{23}$; and have a beneficial effect on the microvasculature of the kidney in patients with diabetes. ${ }^{24}$ In the African American Study of Kidney Disease and Hypertension (AASK), ${ }^{18}$ conducted in 1094 African Americans with hypertension and nondiabetic chronic renal 


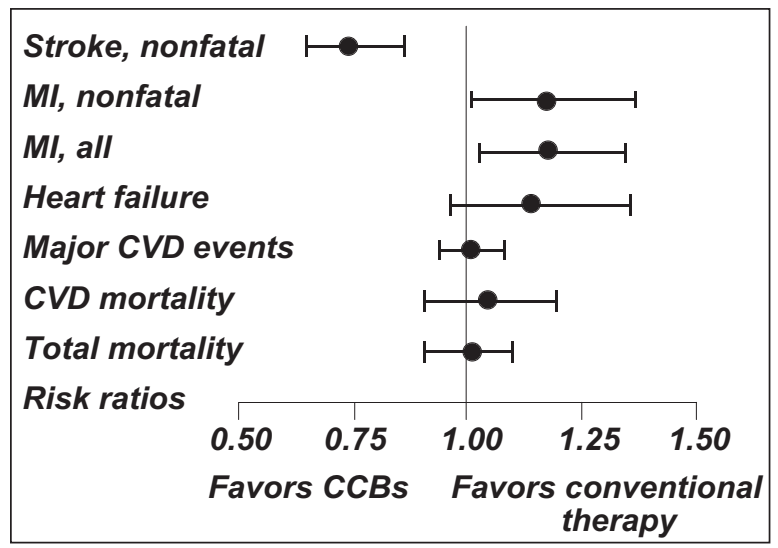

Figure 2. Comparison between effects of calcium channel blocker (CCB)-based therapy and conventional therapy consisting of diuretics or $\beta$ blockers on specific clinical end points. Clinical trial data favored CCBs for stroke $(\mathrm{p}=0.001)$ and conventional therapy for total myocardial infarction (MI) ( $\mathrm{p}=0.036)$; however, stroke reduction more than balanced increased MI. All other clinical outcomes were not significant. CVD=cardiovascular disease; includes data from International Nifedipine GITS study: Intervention as a Goal in Hypertension Treatment (INSIGHT), Nordic Diltiazem study (NORDIL), Swedish Trial in Old Patients with Hypertension-2 study (STOP-2), Verapamil in Hypertension and Atherosclerosis Study (VHAS), and National Intervention Cooperative Study in Elderly Hypertensives study $(\mathrm{NICS}-\mathrm{EH})^{32}$

insufficiency, an antihypertensive regimen based on the ACE inhibitor ramipril was superior to regimens based on the $\beta$ blocker atenolol or the CCB amlodipine besylate for the combined clinical outcome of reduction in glomerular filtration rate by $\geq 50 \%$ from baseline, development of end-stage renal disease, or death. The AASK trial was the first outcome trial to demonstrate a renoprotective effect of ACE inhibitors in African Americans. Through the Heart Outcomes Prevention Evaluation (HOPE) ${ }^{25}$ study, the benefits of ACE inhibitors have been extended to a wide range of high-risk patients, including those without elevated blood pressure. It is important to note that all of the above studies were multiple drug studies; a majority of patients received medications (usually a diuretic) in addition to the ACE inhibitor to achieve goal blood pressures.

Beyond their ability to reduce blood pressure, the beneficial effects of ACE inhibitors are due to their mechanism of action. ACE

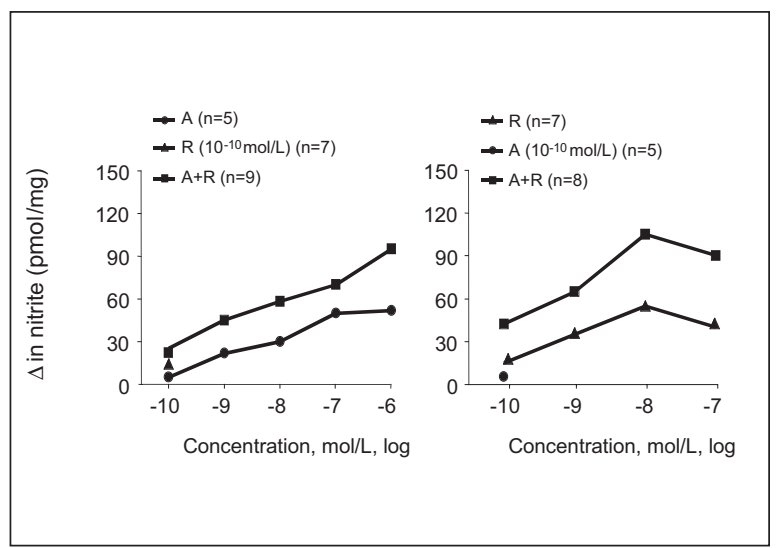

Figure 3. Changes in formation of nitrite (quantified by using the Griess reaction) from coronary microvessels of mongrel dogs in response to ramiprilat $(R)$ and amlodipine besylate $(A)$. Ramiprilat $\left(10^{-10} \mathrm{~mol} / \mathrm{L}\right)$ potentiated the nitrite production induced by increasing concentrations of amlodipine besylate ( $<<0.05$ vs. amlodipine besylate alone); the highest concentration of amlodipine besylate increased nitrite production by $77 \%$ (shown on left). Amlodipine besylate potentiated the effects induced by increasing concentrations of ramiprilat ( $\mathrm{p}<0.05$ vs. ramiprilat alone); the highest concentration of ramipril increased nitrite production by $105 \%$ (shown on right). ${ }^{40}$

inhibition opposes the multiple pathologies that are involved in morbidities that are collectively attributable to activation of the renin-angiotensin-aldosterone system and subsequent increased production of angiotensin II. Angiotensin II promotes vasoconstriction, abnormal smooth muscle cell migration, macrophage activation, and platelet aggregation, and contributes to cardiac and vascular hypertrophy, endothelial dysfunction, and altered renal hemodynamics. Both experimental and clinical evidence suggests that ACE inhibition has beneficial effects by inhibiting vasoconstriction, endothelial dysfunction, the development of atherosclerosis, and thrombus formation. ${ }^{20,26-31}$

\section{CCBs vs. Diuretics in High-Risk Patients}

Because both groups in ACCOMPLISH will receive the ACE inhibitor benazepril $\mathrm{HCl}$, the study is designed to specifically differentiate the combination of an ACE inhibitor/CCB vs. an ACE inhibitor/diuretic. Based on currently available data, neither diuretics nor CCBs can claim to be 
superior in combination with an ACE inhibitor. Although ALLHAT ${ }^{8}$ data suggest that a diuretic is unsurpassed as an initial therapy for hypertension, with fewer episodes of heart failure than a CCB, the trial provided little clarification regarding optimal regimens for combination therapy; even though, on average, ALLHAT participants took two agents, and blood pressure levels $<140 / 90 \mathrm{~mm} \mathrm{Hg}$ were achieved in $>60 \%$ of patients. The combinations being studied in ACCOMPLISH were not utilized in the protocol for ALLHAT.

In an overview of randomized controlled clinical trials ${ }^{5}$ comparing CCBs with other diuretics or $\beta$ blockers, there was no clear winner. Outcomes for stroke were somewhat better with CCB treatment; outcomes for coronary heart disease and heart failure were somewhat better with diuretics; and neither was superior for major CVD events, CVD death, or total mortality. A meta-analysis ${ }^{32}$ of trials including more than 46,000 patients showed similar findings: CCBs demonstrated a lower risk of nonfatal stroke $(-25 \%$; $p=0.004)$ and a higher risk of total MI $(18 \%$; $p=0.052$ ) compared with diuretics or $\beta$ blockers; thus, stroke reduction more than balanced increased MI, although MIs are a more frequent event (Figure 2).

Some data suggest a potential role for CCBs-and amlodipine besylate in particular-in the prevention or regression of atherosclerosis. ${ }^{33,34}$ In the Prospective Randomized Evaluation of the Vascular Effects of Norvasc Trial (PREVENT), ${ }^{35}$ treatment with amlodipine besylate produced benefits compared with placebo, demonstrating marked reductions in CVD events as well as reductions in the progression of carotid atherosclerosis. Further, amlodipine besylate has been shown to have a favorable effect on morbidity and mortality in patients with nonischemic heart failure. ${ }^{36}$

Data from experimental studies support the hypothesis that some of the beneficial effects of ACE inhibitors in the treatment of heart failure may be due to the release of nitric oxide secondary to local generation of kinins. ${ }^{37,38}$ In animal studies, amlodipine besylate-but not nifedipine or dilti- azem-was found to cause a significant, dose-dependent increase in nitrite (the hydration product of nitric oxide) production that was similar in magnitude to that found with ACE inhibitors. ${ }^{39}$ Further, in combination, amlodipine besylate potentiated nitrite production produced by ramiprilat-the active metabolite of the ACE inhibitor ramipril ${ }^{40}$ (Figure 3).

\section{ACCOMPLISH}

The ACCOMPLISH trial will compare amlodipine besylate/benazepril $\mathrm{HCl}$ and benazepril $\mathrm{HCl} /$ hydrochlorothiazide for the clinical outcomes of CVD morbidity and mortality in high-risk hypertensive individuals. It is planned that this prospective, randomized, double-blind trial will enroll 12,600 patients at approximately 650 multinational centers. The duration of this event-driven trial is expected to be about 5 years. The blood pressure target will be $<140 / 90 \mathrm{~mm} \mathrm{Hg}$, but a more aggressive target (i.e., $<130 / 80 \mathrm{~mm} \mathrm{Hg}$ ) for patients with diabetes or renal disease may be utilized at the discretion of the individual investigator.

The trial's primary end point is a composite of CVD death, nonfatal MI, nonfatal stroke, hospitalization for unstable angina, or revascularization procedure. Secondary end points will include individual end points within the primary end point, allcause mortality, hospitalization for heart failure, progression of renal disease, and development of diabetes.

\section{SUMMARY}

Combination therapy is currently the mainstay of treatment for hypertension; however, controlled clinical trials have previously only studied monotherapy or single-agent-based therapy with the use of open-label, add-on agents to achieve blood pressure control. Despite the large amount of data amassed, only a single trial based on this design paradigm has found one class of agents (ARBs) to be superior to another ( $\beta$ blockers) in reducing CVD events. There is little evidence available from completed trials to guide clinicians in the selection of combination antihypertensive regimens for 
high-risk patients. It is time to look at a new paradigm in hypertension trials. The ACCOMPLISH trial is the first large, randomized hypertension clinical trial to compare CVD mortality and morbidity in high-risk hypertensive patients with two different combination regimens: amlodipine besylate/benazepril $\mathrm{HCl}$ vs. benazepril $\mathrm{HCl} / \mathrm{hydrochlorothiazide.}$

\section{REFERENCES}

1 Psaty BM, Smith NL, Siscovick DS, et al. Health outcomes associated with antihypertensive therapies used as first-line agents. A systematic review and meta-analysis. JAMA. 1997;277:739-745.

2 Joint National Committee on Prevention, Detection, Evaluation, and Treatment of High Blood Pressure. The sixth report of the Joint National Committee on Prevention, Detection, Evaluation, and Treatment of High Blood Pressure. Arch Intern Med. 1997;157:2413-2446.

3 Chalmers J, MacMahon S, Mancia G, et al., and the Guidelines Subcommittee of the World Health Organization. 1999 World Health OrganizationInternational Society of Hypertension guidelines for the management of hypertension. Clin Exp Hypertens. 1999; 21:1009-1060.

4 Chobanian AV, Bakris GL, Black HR, et al., and the National High Blood Pressure Education Program Coordinating Committee. The seventh report of the Joint National Committee on Prevention, Detection, Evaluation, and Treatment of High Blood Pressure: the JNC 7 report. JAMA. 2003;289:2560-2572.

5 Neal B, MacMahon S, Chapman N, for the Blood Pressure Lowering Treatment Trialists' Collaboration. Effects of ACE inhibitors, calcium antagonists, and other blood-pressure-lowering drugs: results of prospectively designed overviews of randomised trials. Lancet. 2000;356: 1955-1964.

6 Kjeldsen SE, Westheim AS, Os I. INSIGHT and NORDIL. International Nifedipine GITS study: Intervention as a Goal in Hypertension Treatment. Nordic Diltiazem Study. Lancet. 2000;356:1929-1930.

7 Dahlöf B, Devereux RB, Kjeldsen SE, et al., for the LIFE Study Group. Cardiovascular morbidity and mortality in the Losartan Intervention For Endpoint reduction in hypertension study (LIFE): a randomised trial against atenolol. Lancet. 2002;359:995-1003.

8 The ALLHAT Officers and Coordinators for the ALLHAT Collaborative Research Group. Major outcomes in highrisk hypertensive patients randomized to angiotensin-converting enzyme inhibitor or calcium channel blocker vs diuretic: the Antihypertensive and Lipid-Lowering Treatment to Prevent Heart Attack Trial (ALLHAT). JAMA. 2002;288:2981-2997.

9 Black HR, Elliott WJ, Grandits G, et al. Principal results of the Controlled Onset Verapamil Investigation of Cardiovascular End Points (CONVINCE) trial. JAMA. 2003;289:2073-2082.

10 Psaty BM, Lumley T, Furberg CD, et al. Health outcomes associated with various antihypertensive therapies used as first-line agents: a network meta-analysis. JAMA. 2003;289 :2534-2544.

11 Hansson L, Hedner T, Lund-Johansen P, et al. Randomised trial of effects of calcium antagonists compared with diuretics and beta-blockers on cardiovascular morbidity and mortality in hypertension: the Nordic Diltiazem (NORDIL) study. Lancet. 2000;356:359-365.

12 Hansson L, Lindholm LH, Niskanen L, et al. Effect of angiotensin-converting-enzyme inhibition compared with conventional therapy on cardiovascular morbidity and mortality in hypertension: the Captopril Prevention Project (CAPPP) randomised trial. Lancet. 1999;353:611-616.

13 Lindholm LH, Hansson L, Ekbom T, et al., for the STOP Hypertension-2 Study Group. Comparison of antihypertensive treatments in preventing cardiovascular events in elderly diabetic patients: results from the Swedish Trial in Old Patients with Hypertension-2. J Hypertens. 2000;18: 1671-1675.

14 Brown MJ, Palmer CR, Castaigne A, et al. Morbidity and mortality in patients randomised to double-blind treatment with a long-acting calcium-channel blocker or diuretic in the International Nifedipine GITS study: Intervention as a Goal in Hypertension Treatment (INSIGHT). Lancet. 2000;356:366-372.

15 Jamerson KA, Bakris GL, Douglas JG, et al. Design of the ACCOMPLISH (Avoiding Cardiovascular Events through Combination Therapy in Patients Living with Systolic Hypertension) trial [abstract P-431]. Am J Hypertens. 2003;16:193A.

16 Neutel JM. Low-dose antihypertensive combination therapy: its rationale and role in cardiovascular risk management. Am J Hypertens. 1999;12(8 pt 2):73S-79S.

17 Wing LM, Reid CM, Ryan P, et al., for the Second Australian National Blood Pressure Study Group. A comparison of outcomes with angiotensin-converting-enzyme inhibitors and diuretics for hypertension in the elderly. $\mathrm{N}$ Engl J Med. 2003;348:583-592.

18 Wright JT Jr, Bakris GL, Green T, et al. Effect of blood pressure lowering and antihypertensive drug class on progression of hypertensive kidney disease: results from the AASK trial. JAMA. 2002;288:2421-2431.

19 Maschio G, Marcantoni C, Bernich P. Lessons from large interventional trials on antihypertensive therapy in chronic renal disease. Nephrol Dial Transplant. 2002;17(suppl 11):47-49.

20 Bakris GL. Angiotensin-converting enzyme inhibition to enhance vascular health-clinical and research models. Am J Hypertens. 2001;14(8 pt 2):264S-269S.

21 Bicket DP. Using ACE inhibitors appropriately. Am Fam Physician. 2002;66:461-468.

22 Kshirsagar AV, Joy MS, Hogan SL, et al. Effect of ACE inhibitors in diabetic and nondiabetic chronic renal disease: a systematic overview of randomized placebo-controlled trials. Am J Kidney Dis. 2000;35:695-707.

23 Kirpichnikov D, Sowers JR. Role of ACE inhibitors in treating hypertensive diabetic patients. Curr Diab Rep. 2002;2:251-257.

24 Abbott KC, Bakris GL. Treatment of the diabetic patient: focus on cardiovascular and renal risk reduction. Prog Brain Res. 2002;139:289-298.

25 Heart Outcomes Prevention Evaluation Study Investigators. Effects of ramipril on cardiovascular and microvascular outcomes in people with diabetes mellitus: results of the HOPE study and MICRO-HOPE substudy. Lancet. 2000;355:253-259.

26 Heinig RE. What should the role of ACE inhibitors be in the treatment of diabetes? Lessons from HOPE and MICROHOPE. Diabetes Obes Metab. 2002;4(suppl 1):S19-S25.

27 Halkin A, Keren G. Potential indications for angiotensinconverting enzyme inhibitors in atherosclerotic vascular disease. Am J Med. 2002;112:126-134.

28 Enseleit F, Hurlimann D, Luscher TF. Vascular protective effects of angiotensin converting enzyme inhibitors and their relation to clinical events. J Cardiovasc Pharmacol. 2001;37(suppl 1):S21-S30.

29 Izzo JL Jr. The role of the renin-angiotensin system in vascular health: use of ACE inhibition to improve vascular function. Heart Dis. 2000;2:380-383.

30 Felmeden DC, Lip GY. The renin-angiotensin-aldosterone system and fibrinolysis. J Renin Angiotensin Aldosterone Syst. 2000;1:240-244. 
31 Mancini GB. Role of angiotensin-converting enzyme inhibition in reversal of endothelial dysfunction in coronary artery disease. Am J Med. 1998;105:40S-47S.

32 Opie LH, Schall R. Evidence-based evaluation of calcium channel blockers for hypertension: equality of mortality and cardiovascular risk relative to conventional therapy. $J$ Am Coll Cardiol. 2002;39:315-322.

33 Ferrari R. Angiotensin converting enzyme inhibitor-calcium antagonist combination: an alliance for cardioprotection? J Hypertens Suppl. 1997;15:S109-S117.

34 Messerli FH, Michalewicz L. Cardiac effects of combination therapy. Am J Hypertens. 1997;10(7 pt 2):146S-152S.

35 Preston Mason R. Atheroprotective effects of long-acting dihydropyridine-type calcium channel blockers: evidence from clinical trials and basic scientific research. Cerebrovasc Dis. 2003;16(suppl 3):11-17.

36 Packer M, O'Connor CM, Ghali JK, et al., for the Prospective Randomized Amlodipine Survival Evaluation
Study Group. Effect of amlodipine on morbidity and mortality in severe chronic heart failure. N Engl J Med. 1996; 335:1107-1114

37 Kichuk MR, Zhang X, Oz M, et al. Angiotensin-converting enzyme inhibitors promote nitric oxide production in coronary microvessels from failing explanted human hearts. Am J Cardiol. 1997;80:137A-142A.

38 Zhang X, Scicli GA, Xu X, et al. Role of endothelial kinins in control of coronary nitric oxide production. Hypertension. 1997;30:1105-1111.

39 Zhang X, Hintze TH. Amlodipine releases nitric oxide from canine coronary microvessels: an unexpected mechanism of action of a calcium channel-blocking agent. Circulation. 1998;97:576-580.

40 Zhang X, Xu X, Nasjletti A, et al. Amlodipine enhances NO production induced by an ACE inhibitor through a kinin-mediated mechanism in canine coronary microvessels. J Cardiovasc Pharmacol. 2000;35:195-202. 